This item was submitted to Loughborough's Research Repository by the author.

Items in Figshare are protected by copyright, with all rights reserved, unless otherwise indicated.

\title{
Far-field representation for the vertical force on a floating structure
}

PLEASE CITE THE PUBLISHED VERSION

http://dx.doi.org/10.1017/jfm.2012.449

PUBLISHER

(c) Cambridge University Press

VERSION

VoR (Version of Record)

LICENCE

CC BY-NC-ND 4.0

REPOSITORY RECORD

Mclver, M.. 2019. "Far-field Representation for the Vertical Force on a Floating Structure". figshare. https://hdl.handle.net/2134/11791. 
This item was submitted to Loughborough's Institutional Repository (https://dspace.lboro.ac.uk/) by the author and is made available under the following Creative Commons Licence conditions.

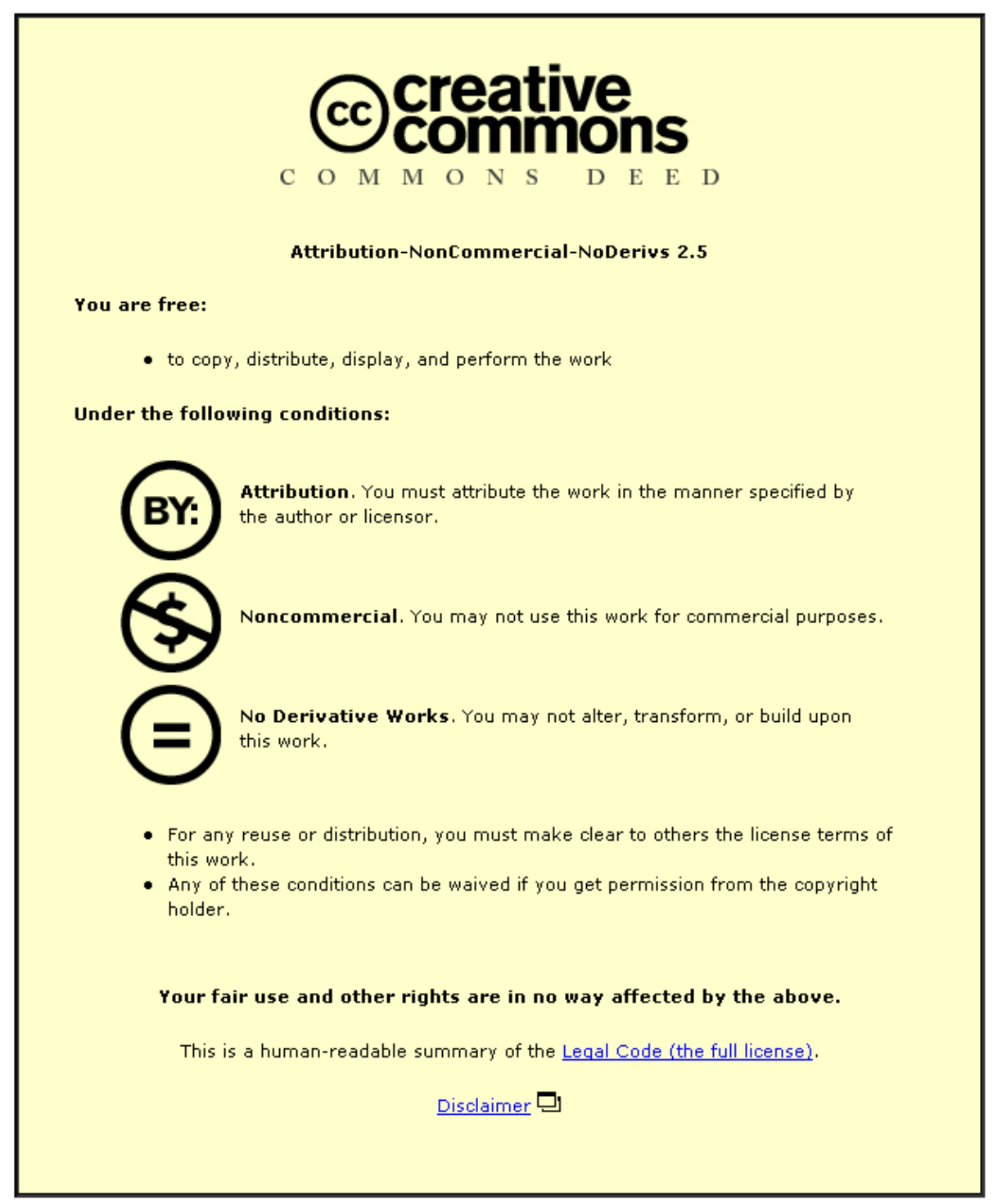

For the full text of this licence, please go to: http://creativecommons.org/licenses/by-nc-nd/2.5/ 


\title{
Far-field representation for the vertical force on a floating structure
}

\author{
M. McIver $\dagger$ \\ Department of Mathematical Sciences, Loughborough University, Loughborough LE11 3TU, UK \\ (Received 4 July 2012; revised 4 September 2012; accepted 7 September 2012; \\ first published online 16 October 2012)
}

Equations are derived that relate the vertical hydrodynamic force on two- and threedimensional structures that are floating in a fluid of infinite depth to the far-field dipole coefficient in the velocity potential. By an application of Green's theorem to the radiation or scattering potential and a suitable test potential, the heave added mass, the heave damping and the vertical exciting force are shown to be expressible in terms of the dipole coefficient in the relevant potential. The results add to the known reciprocity relations, which relate quantities such as the damping and the exciting force to the amplitude of the far-field radiated wave. The expressions are valid at all frequencies, and their high- and low-frequency asymptotics are investigated.

Key words: surface gravity waves, wave scattering, wave-structure interactions

\section{Introduction}

It is well known (Newman 1977) that, when a structure makes small oscillations at angular frequency $\omega$ in a fluid with a free surface, the part of the hydrodynamic force on the body that is in phase with its velocity, the damping, is related to the amplitude of the radiated wave and is non-negative. This is one of many reciprocity relations that have been found that relate near-field and far-field quantities associated with the radiation and scattering of waves by a floating structure, and the known results are summarized in Linton \& McIver (2001). No similar relationship is known for the part of the force on a body that is in phase with the acceleration, the added mass, although Kotik \& Mangulis (1962) have shown that this is related to an integral of the damping over all frequencies, via the Kramers-Kronig relations. The Kramers-Kronig relations were also used by Greenhow (1986) to obtain simple highand low-frequency relationships between the added mass and damping.

The first aim of this work is to show, by the application of Green's theorem to the heave radiation potential and a suitable test potential, that, when a structure makes small oscillations in the vertical direction in a fluid of infinite depth, the total hydrodynamic force it experiences, which is made up of both the heave added mass and the heave damping, is related to the far-field dipole coefficient in the velocity potential. The method used follows that given in McIver \& McIver (2006, 2007) to generate necessary conditions for the existence of motion trapped modes. The resulting expression for the heave force is valid at any frequency and is an extension of the

$†$ Email address for correspondence: m.mciver@lboro.ac.uk 
result derived by Newman (1977), who showed that the force on a body that moves in an unbounded fluid with no free surface is related to the dipole moment in the far field. Far-field representations are given for the vertical force on both two- and three-dimensional structures, and the method is then extended to show that the vertical exciting force on a fixed structure that scatters an incident wave can be written in terms of the coefficient of the far-field vertical dipole in the scattering potential.

Although the expressions for the vertical forces are valid at all frequencies, in general the far-field dipole coefficient needs to be evaluated numerically for any particular structure. This is fairly straightforward at low or moderate frequencies, for structures like a floating semicircular cylinder (Ursell 1949) or a hemisphere (Havelock 1955), where the potential can be written in terms of a multipole expansion everywhere in the fluid. However, numerical calculations are more difficult at high frequencies, and Leppington (1973) developed a method based on matched asymptotic expansions to obtain a high-frequency approximation to the heave potential. His work requires the fluid to be split into a number of regions around the structure and the potential to be expanded differently in each region. This makes it difficult to evaluate the force on the structure by direct integration of the pressure over the body, as the body typically extends into more than one region and so there is not a single form for the potential that can be used. The relationship between the total force and the far-field dipole coefficient that is derived in this work has the advantage that the force is written in terms of the potential in a single region.

Many authors have studied the low-frequency approximation to the hydrodynamic forces on a structure, and much of the work that has been done is summarized in McIver (1994). The method of matched asymptotic expansions may be used in a straightforward way at low frequencies to determine the asymptotic expansion of the force on a structure by direct integration of the potential, as typically the structure is contained within a single region in a fluid and so only one form of the potential is used. However, it will be shown that the expressions for the vertical force that are derived in this work may be combined with the results from direct integration to obtain further terms in the asymptotic expansion of the far-field dipole coefficient at low frequencies.

The results obtained in this work and the other reciprocity relations summarized in Linton \& McIver (2001) are based on the assumption of small-amplitude motion and use linear theory. However, results also exist (Maruo 1960; Newman 1967) that relate the second-order drift force and vertical moment on a structure to integrals that involve the far-field wave amplitude. More recently, Sclavounos (2012) derived expressions for the fully nonlinear wave loading on a structure in terms of the time derivative of the fluid impulse.

\section{The vertical force coefficient}

For simplicity, the theory is given in detail for a single two-dimensional surfacepiercing structure that is symmetric in $x$ and makes small vertical oscillations with angular frequency $\omega$ about its equilibrium position, as illustrated in figure 1. The results for an asymmetric structure, a multi-hulled structure and a three-dimensional structure are presented, but, as the derivation is essentially the same, the details are not given. In addition, expressions are given for the vertical exciting force on two- and three-dimensional structures that are held fixed in waves.

Coordinate axes are chosen so that the $z$-axis points vertically upwards, the mean free surface $(F S)$ coincides with the line $z=0$ and the structure intersects the surface 


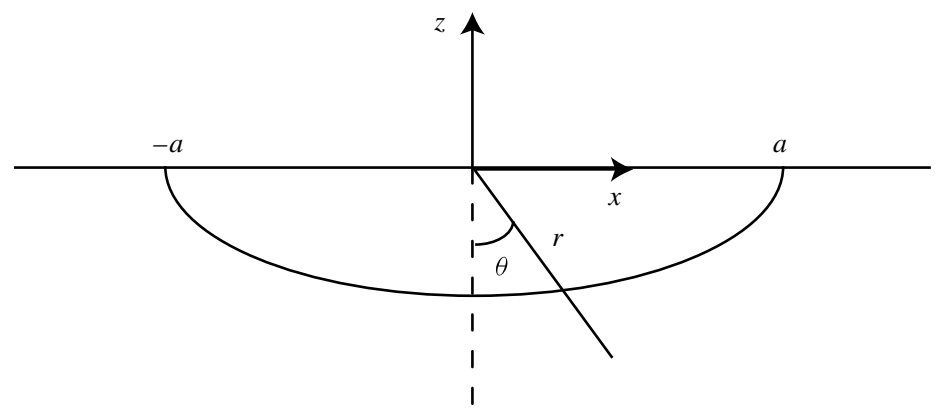

FIGURE 1. Definition sketch.

at $x= \pm a$. Two-dimensional polar coordinates $(r, \theta)$ are defined such that

$$
x=r \sin \theta, \quad z=-r \cos \theta .
$$

The water is assumed to be inviscid and incompressible and the motion irrotational, so the velocity may be written as the gradient of a potential that satisfies Laplace's equation. The motion of the structure and the fluid is assumed to be sufficiently small that linear theory may be used (Newman 1977), and so it is possible to consider a body that oscillates at a single frequency in one mode of motion. Vertical oscillations are governed by the heave radiation potential $\operatorname{Re}\left[\phi(x, z) \mathrm{e}^{-\mathrm{i} \omega t}\right]$, which satisfies

$$
\nabla^{2} \phi=0 \text { in the fluid, }
$$

and the boundary condition

$$
\frac{\partial \phi}{\partial n}=n_{z} \quad \text { on } S,
$$

where $S$ is the structure surface, $\partial / \partial n$ represents the derivative in the direction of the inward normal to $S$ and $n_{z}$ is the component of the inward normal to the body in the $z$-direction. On the mean free surface, $\phi$ satisfies the condition

$$
K \phi-\frac{\partial \phi}{\partial z}=0 \quad \text { on } F S,
$$

where $K=\omega^{2} / g$ and $g$ is the acceleration due to gravity. The potential represents outward propagating waves as $x \rightarrow \pm \infty$ and decays with depth. The boundary value problem for $\phi$ is assumed to possess a unique solution. This has been established for various classes of single-body structures, but non-uniqueness is known to occur for certain multi-hulled two-dimensional structures and three-dimensional structures that possess moon pools. A review of some of the known results on uniqueness and the existence of trapped modes is available in Kuznetsov, Maz'ja \& Vainberg (2002).

Ursell (1968) showed that outside a semicircle that is centred on the mean free surface and contains the structure, $\phi$ may be written as

$$
\phi=a p_{0} G(x, z)+\sum_{n=1}^{\infty} a^{2 n+1} p_{n} \phi_{n}(r, \theta),
$$

where $G$ is the potential due to a wave source at the origin, given by

$$
G=-\psi_{0}^{\infty} \frac{\mathrm{e}^{k z} \cos k x}{k-K} \mathrm{~d} k
$$


(the integral is indented below the pole at $k=K$ ). The potentials

$$
\phi_{n}=\frac{\cos (2 n \theta)}{r^{2 n}}+\frac{K}{2 n-1} \frac{\cos ((2 n-1) \theta)}{r^{2 n-1}}, \quad n=1,2, \ldots,
$$

are the symmetric wave-free potentials that decay as $r \rightarrow \infty$. As the structure is symmetric about the $z$-axis and oscillates in the vertical direction, there is no contribution to $\phi$ from any antisymmetric terms. The coefficients $\left\{p_{n}\right\}$ depend on the geometry of the structure and the wave frequency, and in general must be solved for numerically. The first of these, $p_{0}$, measures the amplitude of the wave radiated to infinity. By writing the source potential in terms of the exponential integral and using the large-argument expansion of this function (Abramowitz \& Stegun 1965), it may be shown that

$$
\phi=-\pi \mathrm{i} a p_{0} \mathrm{e}^{K z+\mathrm{i} K|x|}+\left(\frac{a p_{0}}{K}+K a^{3} p_{1}\right) \frac{\cos \theta}{r}+O\left(\frac{1}{r^{2}}\right) \quad \text { as } r \rightarrow \infty .
$$

For what follows, it is important to note that the dipole coefficient in $\phi$ at large depths does not depend just on the coefficient of the first wave-free potential in (2.5); there is also a contribution from the coefficient of the wave source.

By putting $n=0,-1,-2, \ldots$ in (2.7), a second set of wave-free potentials are generated, which satisfy (2.2) and (2.4) but grow as $r \rightarrow \infty$. The first potential in this set is given by

$$
\phi_{0}=1-K r \cos \theta=1+K z,
$$

and this has the property that

$$
\frac{\partial \phi_{0}}{\partial n}=K n_{z}
$$

on any structure. Following McIver \& McIver (2006), an application of Green's theorem to $\phi$ and $\phi_{0}$ is made in the fluid region that is bounded by a closing surface as $r \rightarrow \infty$, and gives

$$
\int_{\partial D}\left[\phi \frac{\partial \phi_{0}}{\partial n}-\phi_{0} \frac{\partial \phi}{\partial n}\right] \mathrm{d} S=0
$$

where $\partial D$ is the total boundary of the region and $\partial / \partial n$ is the derivative in the outward normal direction to the fluid. As both $\phi$ and $\phi_{0}$ satisfy the free surface condition (2.4), the only contributions to this integral come from the body surface $S$ and the closing surface at infinity. From (2.3)-(2.10) the contribution from the surface of the body is given by

$$
\int_{S}\left[\phi \frac{\partial \phi_{0}}{\partial n}-\phi_{0} \frac{\partial \phi}{\partial n}\right] \mathrm{d} S=K \int_{S} \phi n_{z} \mathrm{~d} S-\int_{S}(1+K z) n_{z} \mathrm{~d} S .
$$

The function $\psi=z+K z^{2} / 2$ satisfies $\nabla^{2} \psi=K$ everywhere, and so the second integral in (2.12) may be evaluated explicitly by an application of the divergence theorem to $\nabla \psi$ in the region contained within the body. As $n_{z}$ is defined to be the $z$-component of the normal that points into the body, (2.12) simplifies to

$$
\int_{S}\left[\phi \frac{\partial \phi_{0}}{\partial n}-\phi_{0} \frac{\partial \phi}{\partial n}\right] \mathrm{d} S=K \int_{S} \phi n_{z} \mathrm{~d} S+K A_{0}-L_{0}
$$


where $L_{0}$ is the water-plane length and $A_{0}$ is the cross-sectional area of the portion of the body below this line. For a single structure that intersects the mean free surface at the two points $x= \pm a$ only, $L_{0}=2 a$.

From (2.9), $\phi_{0}=O(r)$ as $r \rightarrow \infty$ and $\partial \phi_{0} / \partial r=O(1)$, and so there are possible contributions to (2.11) from the interactions between $\phi_{0}$ and any term that is $O(1 / r)$ or larger in $\phi$ on the closing surface at infinity. Therefore, it is necessary to calculate the form of these contributions over a large surface and then let the surface size tend to infinity. It does not matter what the precise shape of the surface is, and so it is sensible to choose it to be a large rectangle when considering the interaction between $\phi_{0}$ and the wave-like term in $\phi$ and a large semicircle of radius $R$ when considering the interaction between $\phi_{0}$ and the dipole in $\phi$.

The propagating wave potential $\phi_{w}$ is defined by

$$
\phi_{w}=\mathrm{e}^{K z+\mathrm{i} K|x|} .
$$

Evaluation of the contribution to the integral in (2.11) from the interaction between $\phi_{w}$ and $\phi_{0}$ on any line $x=L>0,-\infty<z<0$, gives

$$
\int_{-\infty}^{0}\left[\phi_{w} \frac{\partial \phi_{0}}{\partial x}-\phi_{0} \frac{\partial \phi_{w}}{\partial x}\right]_{x=L} \mathrm{~d} z=-\mathrm{i} K \mathrm{e}^{\mathrm{i} K L} \int_{-\infty}^{0}(1+K z) \mathrm{e}^{K z} \mathrm{~d} z=0,
$$

after integration by parts. A similar argument shows that there is no contribution to (2.11) from the interaction between $\phi_{w}$ and $\phi_{0}$ on any line $x=-L<0$ and, as $\phi_{w}$ decays exponentially as $z \rightarrow-\infty$ while $\phi_{0}$ grows only algebraically, there is no contribution to this integral from a closing line at large depths.

The contribution to the integral in (2.11) from the interaction between the dipole potential

$$
\phi_{d}=\frac{\cos \theta}{r}
$$

and $\phi_{0}$ over the surface $r=R, R \rightarrow \infty$, is given by

$$
\begin{aligned}
& \lim _{R \rightarrow \infty}\left[\int_{-\pi / 2}^{\pi / 2}\left(\phi_{d} \frac{\partial \phi_{0}}{\partial r}-\phi_{0} \frac{\partial \phi_{d}}{\partial r}\right) R \mathrm{~d} \theta\right] \\
& \quad=\lim _{R \rightarrow \infty}\left[\int_{-\pi / 2}^{\pi / 2}\left(\frac{\cos \theta}{R}(-K \cos \theta)-(1-K R \cos \theta)\left(-\frac{\cos \theta}{R^{2}}\right)\right) R \mathrm{~d} \theta\right] \\
& \quad=\lim _{R \rightarrow \infty}\left[-K \pi+O\left(\frac{1}{R}\right)\right]=-K \pi .
\end{aligned}
$$

A combination of (2.8), (2.15) and (2.17) shows that the contribution to (2.11) from the closing surface at infinity $S_{\infty}$ is given by

$$
\int_{S_{\infty}}\left[\phi \frac{\partial \phi_{0}}{\partial n}-\phi_{0} \frac{\partial \phi}{\partial n}\right] \mathrm{d} S=-\pi a\left[p_{0}+(K a)^{2} p_{1}\right] .
$$

Substitution of (2.13) and (2.18) into (2.11) gives, after some rearrangement,

$$
F_{2}=\frac{a_{33}}{\rho A_{0}}+\frac{\mathrm{i} b_{33}}{\omega \rho A_{0}}=\frac{1}{A_{0}} \int_{S} \phi n_{z} \mathrm{~d} S=\frac{L_{0}}{K A_{0}}-1+\frac{\pi a p_{0}}{K A_{0}}+\frac{\pi K a^{3} p_{1}}{A_{0}} .
$$

The complex quantity $F_{2}$ is the total non-dimensional heave force on the structure, and (2.19) shows that it may be written in terms of the complex amplitude of the radiated 
wave, the coefficient of the first decaying wave-free potential, the wavenumber $K$ and some of the geometric parameters of the body. The real part of $F_{2}, a_{33} / \rho A_{0}$, is the nondimensional added mass, and the imaginary part, $b_{33} / \omega \rho A_{0}$, is the non-dimensional damping, where $\rho$ is the fluid density and the notation used follows that given in Linton \& McIver (2001). The coefficients $p_{0}$ and $p_{1}$ depend on the wavenumber $K$ and the geometry of the structure, and in general are complex and solved for numerically.

The damping is known to be expressible in terms of the magnitude of the radiated wave amplitude, and the precise relationship may be obtained by an application of Green's theorem to $\phi$ and its complex conjugate $\bar{\phi}$. With the notation used in this paper, this reduces to

$$
\operatorname{Im}\left[\int_{S} \phi n_{z} \mathrm{~d} S\right]=\frac{1}{2 \mathrm{i}} \int_{S}(\phi-\bar{\phi}) n_{z} \mathrm{~d} S=\pi^{2} a^{2}\left|p_{0}\right|^{2} .
$$

Substitution of (2.20) into the imaginary part of (2.19) shows, after some rearrangement, that $\operatorname{Im}\left[p_{1}\right]$ may be written in terms of $p_{0}$ for any structure as

$$
\operatorname{Im}\left[p_{1}\right]=\frac{\pi}{K a}\left|p_{0}\right|^{2}-\frac{1}{(K a)^{2}} \operatorname{Im}\left[p_{0}\right] .
$$

For some structures, such as the semicircular cylinder, the heave potential may be expressed in terms of a multipole expansion at all points in the fluid. The multipole coefficients in general satisfy an infinite system of equations, which must be solved numerically. Direct integration of the potential over such a body leads to the added mass and damping being expressed as infinite sums over all the multipole coefficients. In this case, (2.19) provides a numerical check on the results, which may be particularly useful at high frequencies where numerical calculations are not so easy to make. In addition, the expression in (2.21) may be used to provide a numerical check on the relationship between the first two coefficients in the expansion.

The analysis was performed for a two-dimensional symmetric structure that intersects the free surface of an infinite-depth fluid at two points only. It is straightforward to show that the symmetry requirement is not necessary, because, although the far-field expression for the heave radiation potential for an asymmetric structure includes a horizontal wave dipole and antisymmetric wave-free potentials in addition to the symmetric potentials in (2.5), these extra terms do not contribute to the far-field integral in (2.11). The expression (2.19) also holds for the total vertical force on a multi-body structure that moves as a single entity. In this case, the parameter $a$ is chosen to be a length scale associated with the structure, $L_{0}$ is the sum of the water-plane lengths associated with each body and $A_{0}$ is the corresponding sum of the cross-sectional areas. However, the determination of the coefficients $p_{0}$ and $p_{1}$ in (2.19) requires the far-field expansion of the heave potential in terms of potentials that have a single singular point. Although this is possible for a multi-body structure, it may be more convenient to express the heave potential in terms of separate sets of singularities with singular points inside each body before performing the analysis.

A similar analysis may be performed if the body is submerged, but, although (2.5) is a valid representation of the heave potential for a symmetric body outside a semicircle centred on the free surface and that contains the structure, it is more convenient to express the potential in terms of a submerged vertical wave dipole and submerged wave-free potentials, all with singular points inside the body. The analysis may also be performed if the water has finite depth, but, in this case, the force on the structure 
is given in terms of a limit of an integral of the potential over the sea bed after cancellation is made with contributions from the closing surfaces as $x \rightarrow \pm \infty$.

The far-field representation of the heave potential for an axisymmetric surfacepiercing structure in three dimensions and infinite depth is given by

$$
\phi=a^{2} q_{0} \oint_{0}^{\infty} \frac{k \mathrm{e}^{k z} \mathrm{~J}_{0}(k r \sin \theta)}{k-K} \mathrm{~d} k+\sum_{n=1}^{\infty} a^{2 n+2} q_{n}\left[\frac{P_{2 n}(\cos \theta)}{r^{2 n+1}}+\frac{K}{2 n} \frac{P_{2 n-1}(\cos \theta)}{r^{2 n}}\right],
$$

where $\mathbf{J}_{0}$ is the zero-order Bessel function, $P_{n}(\cos \theta)$ are the Legendre polynomials and the coefficients $\left\{q_{n}\right\}$ depend on the geometry of the structure and the wave frequency. The $z$-axis is chosen as in the two-dimensional case and $r$ and $\theta$ are now two of the spherical polar coordinates, defined by $r=\left(x^{2}+y^{2}+z^{2}\right)^{1 / 2}$ and $z=-r \cos \theta$. The parameter $a$ is chosen to be the radius of the largest circle with which the structure intersects the free surface. A similar analysis to that performed for the twodimensional structure shows that the total non-dimensional heave force on the structure is given by

$$
F_{3}=\frac{a_{33}}{\rho V_{0}}+\frac{\mathrm{i} b_{33}}{\omega \rho V_{0}}=\frac{1}{V_{0}} \int_{S} \phi n_{z} \mathrm{~d} S=\frac{W_{0}}{K V_{0}}-1+\frac{2 \pi a^{2} q_{0}}{K V_{0}}+\frac{\pi K a^{4} q_{1}}{V_{0}},
$$

where $V_{0}$ is the volume of the part of the structure contained in $z<0$ and $W_{0}$ is its water-plane area. For an axisymmetric structure that intersects the mean free surface in only one circle, $W_{0}=\pi a^{2}$. As in the two-dimensional case, (2.23) may be shown to be valid for a non-axisymmetric structure, where $a^{2} q_{0}$ and $a^{4} q_{1}$ are the coefficients of the axisymmetric source and the first axisymmetric wave-free potential, respectively, in the far-field representation of $\phi$.

The expressions in (2.19) and (2.23) are valid for all frequencies, although the coefficients themselves depend on frequency. They are extensions of the result derived by Newman (1977), which states that the force on a body that moves in an unbounded fluid with no free surface is related to the dipole moment in the far field. A similar procedure may be used to obtain expressions for the vertical exciting force on a structure that is held fixed in an incident wave field. In this case, the scattering potential $\phi_{s}$ has zero normal derivative on the structure, and so the expressions for the two- and three-dimensional vertical exciting forces, $X_{2}$ and $X_{3}$, just contain the terms that involve the coefficients in the far-field expansion of $\phi_{s}$, namely

$$
X_{2}=\frac{a}{A_{0}} \int_{S} \phi_{s} n_{z} \mathrm{~d} S=\frac{\pi a p_{0}^{\prime}}{K A_{0}}+\frac{\pi K a^{3} p_{1}^{\prime}}{A_{0}}
$$

and

$$
X_{3}=\frac{a}{V_{0}} \int_{S} \phi_{s} n_{z} \mathrm{~d} S=\frac{2 \pi a^{2} q_{0}^{\prime}}{K V_{0}}+\frac{\pi K a^{4} q_{1}^{\prime}}{V_{0}} .
$$

Here the incident wave is assumed to propagate in the positive $x$-direction, so $\phi_{s}$ can be written as

$$
\phi_{s}=\mathrm{e}^{\mathrm{i} K x+K z}+\phi_{D},
$$

where $\phi_{D}$ is the part of $\phi_{s}$ that remains once the incident wave has been subtracted out. The parameters $\left\{p_{0}^{\prime}, a^{2} p_{1}^{\prime}\right\}$ and $\left\{a q_{0}^{\prime}, a^{3} q_{1}^{\prime}\right\}$ are the coefficients of the wave source and the first wave-free potential in the symmetric or axisymmetric component of $\phi_{D}$. 
Expressions (2.24) and (2.25) may also be used to determine the force on a fixed structure due to a sloshing trapped mode (if one exists), in which case $\phi_{s}$ represents the trapped mode potential and the coefficient of the wave-like term, $p_{0}^{\prime}$ or $q_{0}^{\prime}$, is automatically zero. This last expression was derived by FitzGerald \& McIver (2010) and then used to generate structures that support passive trapped modes.

In general it is only possible to evaluate the coefficients in the expansion of the potential numerically, but information about their behaviour at high and low frequencies may be obtained from the method of matched asymptotic expansions (Leppington 1973). This method requires the fluid domain to be split into a number of separate regions and the potential to be expanded differently in each region and then matched in an appropriate way. In order to determine the force on the structure directly, it is necessary to integrate the potential over the whole body. This is complicated by the fact that the body may be contained within more than one region and so care must be taken when deciding which expansion for the potential should be used. The advantage of the formulae in (2.19) and (2.23)-(2.25) is that they only require the coefficients in the expansion of the potential in the far field.

Leppington (1973) showed that, if a single heaving two-dimensional body intersects the free surface at right angles with a non-zero radius of curvature, then $p_{0}=$ $O\left(1 /(K a)^{2}\right)$ and $K a p_{1}=O(1)$ as $K a \rightarrow \infty$. So from (2.19) the term involving $p_{0}$ does not contribute to the leading-order high-frequency terms in the heave force for such a body. The expansion of $K a p_{1}$ to $O(1 / K a)$ comes from the solution of the first two boundary value problems, which arise when a Taylor series expansion of the free surface condition (2.4) is made in powers of $1 / K a$. It may be shown that

$$
K a p_{1}=p_{\infty}+\frac{1}{K a}\left[-\frac{2}{\pi}-\frac{1}{\pi a} \int_{F S}\left(\frac{\partial \phi_{\infty}}{\partial z}\right)^{2} \mathrm{~d} x\right]+O\left(\frac{1}{(K a)^{2}}\right) \quad \text { as } K a \rightarrow \infty,
$$

where $\phi_{\infty}$ is the infinite-frequency heave potential, which satisfies (2.3), is zero on the free surface and has the far-field expansion

$$
\phi_{\infty}=a^{2} p_{\infty} \frac{\cos \theta}{r} \quad \text { as } r \rightarrow \infty .
$$

(The representation of the $O(1 / K a)$ term in (2.27) in terms of $\phi_{\infty}$ is obtained from an application of Green's theorem to $\phi_{\infty}-z$ and the second potential in the Taylor series expansion.) Substitution of (2.27) into (2.19) gives

$$
F_{2}=\frac{\pi a^{2} p_{\infty}}{A_{0}}-1-\frac{1}{K A_{0}} \int_{F S}\left(\frac{\partial \phi_{\infty}}{\partial z}\right)^{2} \mathrm{~d} x+O\left(\frac{1}{(K a)^{2}}\right) \quad \text { as } K a \rightarrow \infty,
$$

which agrees with the result of Rhodes-Robinson (1970), who calculated the highfrequency asymptotics of the force on a cylinder in finite depth by direct integration.

Several authors have worked on the calculation of hydrodynamic forces at low frequencies and much of this is summarized in McIver (1994). In this limit, the dominant term in the expansion of the heave potential in the far field is the wave source. More precisely, in two dimensions, $p_{0}=O(1)$ and $(K a)^{2} p_{1}=o(1)$ as $K a \rightarrow 0$. However, the heave added mass for a two-dimensional surface-piercing structure in fluid of infinite depth has a logarithmic singularity at $K a=0$. McIver showed that it is possible to obtain an expansion of the heave force on a structure, accurate to $O(1)$, by just knowing the leading-order term in $p_{0}$ as $K a \rightarrow 0$. Thus (2.19) provides a mechanism by which the first correction to the dipole coefficient at low frequency may be determined, without having to solve a higher-order problem in the matched 
asymptotic procedure. In particular, Ursell (1964) show that the non-dimensional heave force on a two-dimensional semicircular cylinder is given by

$$
F_{2}=\frac{8}{\pi^{2}}\left(-\ln K a+\frac{3}{2}-2 \ln 2-\gamma\right)+\frac{8 \mathrm{i}}{\pi}+o(1) \quad \text { as } K a \rightarrow 0,
$$

where $\gamma$ is Euler's constant. Substitution of (2.30) into (2.19) shows, after some rearrangement, that the coefficient of the far-field dipole for the semicircular cylinder is given by

$$
\begin{aligned}
p_{0}+(K a)^{2} p_{1}=- & \frac{2}{\pi}+K a\left[-\frac{4 \ln K a}{\pi^{2}}+\frac{1}{2}+\frac{6}{\pi^{2}}-\frac{8 \ln 2}{\pi^{2}}-\frac{4 \gamma}{\pi^{2}}+\frac{4 \mathrm{i}}{\pi}\right]+o(K a) \\
& \quad \text { as } K a \rightarrow 0 .
\end{aligned}
$$

As $p_{0}=O(1)$ and $(K a)^{2} p_{1}=o(1)$ as $K a \rightarrow 0$, the leading-order term in this expression gives $p_{0}=-2 / \pi$, which is consistent with the leading-order amplitude of the radiated wave obtained from the method of matched asymptotic expansions. The next term on the right-hand side of (2.31) potentially contributes to both the correction term in $p_{0}$ and the leading-order term in $(K a)^{2} p_{1}$.

\section{Conclusion}

In this work, expressions for the vertical hydrodynamic forces on a floating structure, in terms of the dipole coefficient in the expansion of the potential in the far field, have been derived. The method used relies on the existence of the harmonic potential $\phi_{0}$, which satisfies both the free surface boundary condition (2.4) and the condition $\partial \phi_{0} / \partial n \propto n_{z}$ on any structure. Unfortunately, this means that a similar approach cannot be used to determine expressions for the horizontal forces and moments on a structure. In two dimensions, the lowest growing antisymmetric wave-free potential that satisfies the free surface boundary condition is

$$
\phi_{a}=x(1+K z)
$$

but this does not in general satisfy $\partial \phi_{a} / \partial n \propto n_{x}$ on a structure and so cannot be used in an application of Green's theorem to obtain the horizontal force on an arbitrary structure.

The resulting expression for the vertical heave force was investigated at high and low frequencies. In particular, it was shown that at low frequencies the far-field dipole coefficient in the potential, accurate to $O(K a)$, may be obtained without the need to solve for it directly to that order.

\section{REFERENCES}

Abramowitz, M. \& Stegun, I. A. 1965 Handbook of Mathematical Functions. Dover.

FitzGerald, C. J. \& MCIVER, P. 2010 Passive trapped modes in the water-wave problem for a floating structure. J. Fluid Mech. 657, 456-477.

GREenHOW, M. 1986 High- and low-frequency asymptotic consequences of the Kramers-Kronig relations. J. Engng Maths 20, 293-306.

Havelock, T. H. 1955 Waves due to a sphere making periodic heaving oscillations. Proc. R. Soc. Lond. A 231, 1-7.

Kotik, J. \& Mangulis, V. 1962 On the Kramers-Kronig relations for ship motions. Intl Shipbuilding Prog. 9 (97), 361-368.

Kuznetsov, N., MaZ'JA, V. \& VAinberg, B. 2002 Linear Water Waves - A Mathematical Approach. Cambridge University Press. 
Leppington, F. G. 1973 On the radiation and scattering of short surface waves. Part 2. J. Fluid Mech. 59 (1), 129-146.

Linton, C. M. \& MCIVER, P. 2001 Handbook of Mathematical Techniques for Wave/Structure Interactions. Chapman \& Hall/CRC.

Maruo, H. 1960 The drift of a body floating on waves. J. Ship Res. 4, 1-10.

MCIVER, P. 1994 Low-frequency asymptotics of hydrodynamic forces on fixed and floating structures. In Ocean Waves Engineering (ed. M. Rahman), pp. 1-49. Computational Mechanics Publications.

McIVer, P. \& McIVer, M. 2006 Trapped modes in the water-wave problem for a freely floating structure. J. Fluid Mech. 558, 53-67.

MCIVER, P. \& MCIVER, M. 2007 Motion trapping structures in the three-dimensional water-wave problem. J. Engng Maths 58, 67-75.

Newman, J. N. 1967 The drift force and moment on ships in waves. J. Ship Res. 6, 10-17.

Newman, J. N. 1977 Marine Hydrodynamics. MIT Press.

RHODES-RoBinson, P. F. 1970 On the short-wave asymptotic motion due to a cylinder heaving on water of finite depth. I. Proc. Camb. Phil. Soc. 67, 423-444.

SClavounos, P. D. 2012 Nonlinear impulse of ocean waves on floating bodies. J. Fluid Mech. 697, 316-335.

URSELL, F. 1949 On the heaving motion of a circular cylinder on the surface of a fluid. Q. J. Mech. Appl. Maths 2, 218-231.

UrSell, F. 1964 The decay of the free motion of a floating body. J. Fluid Mech. 19, 305-319.

Ursell, F. 1968 The expansion of water-wave potentials at great distances. Proc. Camb. Phil. Soc. 64, 811-826. 